\title{
Development of Anti-fouling Coating Using in Marine Environment
}

\author{
Chen Liu \\ Environmental Protection and Energy Saving Center, China Waterborne Transport Research Institute, Beijing, China
}

\section{Email address:}

liuchen@wit.ac.cn

\section{To cite this article:}

Chen Liu. Development of Anti-fouling Coating Using in Marine Environment. International Journal of Environmental Monitoring and Analysis. Vol. 3, No. 5, 2015, pp. 373-376. doi: 10.11648/j.ijema.20150305.30

\begin{abstract}
The marine organism attaching to the ship hull would slow down the ship and increasing fuel consumption. In order to prevent the problem, anti-fouling paints are used to coat the bottoms of ships. At the same time, the harmful environmental effects of these paints such as tributyltin have been recognized. The International Convention on the Control of Harmful Anti-fouling Systems on Ships was adopted by the IMO in 2001 to prohibit the use of harmful organotins in anti-fouling paints used on ships. As the invention entered into force internationally, the most important work is to develop new material to replace the traditional coating. In this work, we summarize the development of anti-fouling paints all over the world and introduce the progress of the latest research.
\end{abstract}

Keywords: Anti-fouling Coating, Invention, Marine Environment

\section{Introduction}

The marine organism attaching to the ship hull would slow down the ship and increasing fuel consumption. In order to prevent the problem, anti-fouling paints are used to coat the bottom of ships. In order to solve this problem, the researchers used a variety of methods, and the most effective way is to use coating containing toxic substances such as three tributyltin. The anti-fouling paint which contains three TBT has good killing effect, and it can release slowly from the surface of the hull so that they could use for 5 years. At the same time, the harmful environmental effects of these paints have been recognized. The researchers found that the TBT could "leach" into the sea water and persist in the water. They could kill sea-life, harming the environment and even enter to the food chain. Thus, the International Convention on the Control of Harmful Anti-fouling Systems on Ships was adopted by the IMO in 2001 to prohibit the use of harmful organotins in anti-fouling paints used on ships.

As the implementation of the Convention, researchers began to develop new anti-fouling paint. In this study, the traditional anti-fouling coatings and the latest research progress are summarized respectively.

\section{Development of Traditional Anti-fouling Coatings}

As the invention entered into force internationally, the most important work is to develop new material to replace the traditional coating.

\subsection{Tin-free Self-polishing Anti-fouling Coatings}

According to the antipollution principle of organic tin self polishing anti-fouling paint, the tin-free self-polishing anti-fouling coating was made. This kind of paint is based on acrylic, which can afford anti-fouling effect. Since the effect is far worse than the tin self-polishing anti-fouling paint, to combine other new materials for improving anti-fouling effect is necessary. The toxicity of improved paint is far less than the tin self-polishing anti-fouling paint, and it could meet the needs of environmental protection and anti-fouling properties. Thus it becomes the mainstream products in anti-fouling paint for many years [1]. This kind of paint contains copper and other metals which could lead to "the black pollution" and could be harmful to marine environment, so it is strictly limited by the European Union. 


\subsection{Low Surface Energy Anti-fouling Coatings}

Low surface-energy anti-fouling paint has very low surface-energy, making it difficult for marine fouling organisms to attach to the hull. Such anti-fouling paint is divided into two types including silicone-based and contains fluoride [2]. In 1972, American researcher first developed silicone-based anti-fouling paint successfully and got the patent. The anti-fouling valid of this material was up to 2-3 years. Although surface energy of silicone is low, its adhesion is weak. So it is necessary to improve the enhancing adhesion of the silicone in future. Fluorocarbon resin has strong weather resistance, water and oil resistance, stain resistance, chemical resistance, solvent resistance etc, thus it is a kind of excellent material for super weather-resistant, anticorrosive and architectural paint [3]. Organic fluoropolymer was applied in the US military at first, and DuPont and $3 \mathrm{M}$ invent the Teflon (PTFE) and Polytrifluorochloroethylene (PCTFE) in 1860s. Afterwards, Fluorine paint has exhibited superior performance in the low surface energy anti-fouling paint, and it obtained widespread concern.

\subsubsection{Modified Organic Silicone Anti-fouling Coatings}

Silicone resin series polymer has special skeleton for the unique function for silicon resin polymers, such as low surface energy and low elastic modulus. It is difficult for marine life to attach because of its low surface tension. So silicone resin polymer seek to be the rich for linear, elastic, fluid skeleton and there is adequate the active side group on surface to decrease the surface energy [4]. Silicone could not stop marine biofouling, but it could reduce the adhesive strength. At the same time, exudation of oil could cut down the marine fouling and promote marine life off with the scouring effect of sea water [5]. Qiu et al. made polysiloxance with tin through the process of inducing organic tin compounds with strong biological activity to polysiloxane molecules. This method solves the volatility problem of tin compounds and decreases the toxicity of the polymer [6]. Silicone anti fouling paint has experienced the development process from silicone rubber to modified silicone resin since $1960 \mathrm{~s}$. Now, researchers mainly use silicone polymer, modified silicone resin and pigment filler reinforced resin to improve performance of anti-fouling coatings for low surface energy.

\subsubsection{Modified Organic Fluorine Anti-fouling Coatings}

The durability and resistance to chemicals of fluoride containing polymer is excellent, but the price is expensive. So researchers modify them to get good anti-fouling coatings Organic fluorine polymer was first developed in the United States in 1930s for the needs of the military industry. In 1938, the United States DUPONT developed PTFE and the non adhesive coating was invented. Then the Pennwalt and Elf Atochem company of the United States synthesised semi-crystalline poly two fluoride paint which named Kynar500. But this paint could not be cured at room temperature and has high sintering temperature. Also, conventional organic solvent is difficult to dissolve this paint.
In 1982, Asahi company research and development fluorocarbon coatings. This kind of paint has a high gloss and transparency, soluble in conventional organic solvents, and can be cured at room temperature. The appearance of this kind of fluorocarbon coatings is greatly expanded the application of fluorocarbon coatings.

\subsubsection{Fluorine Silicone Resin Anti-fouling Coatings}

The mechanical property of silicone resin is poor, and is not resistant to organic solvents, but the flexibility is good. The fluorine resin has excellent properties, and the side groups can improve the solvent resistance and improve the surface properties, but the price is very expensive. Therefore, researchers have developed a new type of anti-fouling coating which have both advantages.

Martinelli et al. Synthesised resin coating containing silicon and fluorine. The coating is hydrophobic and oleophobic properties and the release rate of ulva is higher than $80 \%$ which showed good anti-fouling effect [7].

Chen et al. modified acrylic resin using organic silicon and organic fluorine monomer. The result showed that when the content of silicon monomer is $9 \%$, the surface tension of the coating is higher, which has better anti fouling effect [8].

\subsection{Conductive Anti-fouling Coating}

Conductive anti-fouling coating is using conductivity of the system, electrolyze seawater, hypochlorite produced and then prevent the pollution. One method is coat the boat with a layer of conductive polymer, then the boat is cathode and the Conductive coating is anode. Through the micro current, $\mathrm{ClO}^{-}$layer formed at the surface of coat and So as to achieve the effect of anti fouling. Another method is using doped conductive polymer materials to coat the boat directly without electric current.

Japan Mitsubishi heavy coated the bottom of boat about $100 \mathrm{~m}^{3}$ in 1990 , and there was no marine biofouling after 3 months. Wang et al. produced a conducting polyaniline coating, the coating had the special vulnerability and the anti-fouling performance can be maintained for $6 \sim 9$ months [9]. This technique is a kind of environment-friendly anti-fouling technology, but is easily affected by environmental factors, thus failed to promote the use of.

\subsection{Marine Anti-fouling Coatings}

Marine organisms have natural anti-fouling ability such as seaweed, shellfish, dolphin, whale and others. They live in the marine environment for all the time but few marine organisms attach on them. From the study, researchers found that coral and other marine organisms can produce anti-fouling properties by itself, so human could use the extract of such biological to make anti-fouling paint.

Some researchers studied the extract from coral. After the separation, they got 4 kinds of open loop steroids compounds which could prevent barnacles fouling effectively [10]. Since the high activity and without detriment to other larval growth and development, the extract of coral could be environment friendly anti-fouling agent. Qian et al. studied important AF 
compounds discovered from a variety of organisms from 2004 until mid 2009. They discussed trends in the discovery of AF compounds and summarized updating scientists and engineers on the current challenges facing AF research [11]. Although there are already some achievement on this kind of anti-fouling paint, the products are high cost, short-term effect and anti-fouling effect is not ideal, could not meet the requirement.

\section{Progress of Latest Research}

\subsection{Silicate Anti-fouling Coatings}

Silicate anti-fouling paint is one kind of anti-fouling agent of soluble alkali silicates, which may form strong alkaline region between the hull surface and sea areas of the paint in contact. The suitable habitat $\mathrm{pH}$ for marine fouling organisms is 7.5-8.0 in slightly alkaline seawater, and it is not fit for them in alkali or acid environment. Silicate could become the anti-fouling agent (such as zeolites, crystallization alumina silicate with containing water), and its anti-fouling mechanism may be the ion exchange or molecular sieve effect. During the process, ion exchange happens between silicate and $\mathrm{H}^{+}$which comes from seawater. Then anti-fouling agent is released and anti-fouling function is achieved.

This way is not only the feature for cheap, efficient, no environmental hazards, but also could not be affected by climate change and the marine environment. The Institute of Chemical Technology of Qingdao Ocean made the non-toxic anti-fouling paint powder which was based on alkaline silicate and other additives, and it could be easy to carry and could be saved for 2 years. At the same time, the powder has a good anticorrosive effect after mixed with resin, and its anti-fouling deadline could be more than two years [12].

\subsection{Capsaicin Anti-fouling Agent}

Capsaicin is separated from pepper fruit. It was named by Thresh in 1876 and its structure was determined by Nelson in 1919. It is the main substance of spicy flavor in chili and it is the stable alkaloid. Also, it has the function of antibacterial and could prevent the growth of marine organisms. So far, domestic and foreign researchers have done a lot of research for spicy type of natural anti-fouling agent [13]. Anti-fouling paint with capsaicin achieves the purpose of anti-fouling by driving effect. The effective components of it could be released slowly through release control technology and achieve long-term and safe anti-fouling effect.

Fischer used pepper derivative for anti-fouling agent (e.g. red capsaicin or oleoresin capsaicin) and added it to an ordinary waterproof paint to synthesize the anti-fouling paint which could prevent marine organisms fouling [14]. Yan et al. synthesized three acrylamide compounds derived from capsaicin. They found that all the three compounds possessed good antibacterial activities with MIC value of BMA. These compounds were also studied as anti-fouling agent in anti-fouling paint, and test panels immersed for 186 days was almost free of marine organisms [15]. The result showed that it could prevent the adhesion of marine organisms.

\subsection{Isothiazolinone Anti-fouling Coatings}

Isothiazolone derivative is a general term for a class of derivatives which is widely used as the environment friendly anti-fouling agent. In the 1990s, American company of Rohm\&Haas developed 4,5-Dichloro-2-octyl-isothiazolone successfully and then put it to the market. Related studies showed that the DCOI is a kind of environmental friendly marine anti-fouling agent. This result won the Presidential Green Chemistry Challenge award and the chemical environment prize in 1996 and 1997 respectively.

Anti-fouling mechanism of this kind of compounds is to form hydrogen bonds through the base effect between the active part on the heterocyclic and protein of organisms. Then it destructs the structure of DNA and leads to cell death due to loss of replicative capacity [16]. It can be degraded rapidly in seawater, and it does not form biological accumulation in the marine environment and also no influence to ecological environment and human health consequences. But water solubility of this kind of compounds is poor, and it is not easy to make preparation. Therefore, further work is needed to improve the performance, which is suitable for all kinds of conditions.

\subsection{Nano Anti-fouling Coatings}

The introduction of nano particles could improve the rheology, coating adhesion, coating hardness, smoothness and anti-aging properties for paint, thus it is the one important direction of anti-fouling agent development. Microencapsulation technology is an efficient anti-fouling nano technology, which using a kind of water-soluble resin material to make the anti-fouling agent nano powder to form particle, then formulate in paint. In practical applications, the microcapsules could be dissolved by sea water gradually, and the anti-fouling agent is released slowly and effectively so as to achieve a stable anti-fouling effect. Microencapsulated anti-fouling agent has longer period anti-fouling efficiency, better anti-fouling effect and could reduce environmental impact.

Micro-encapsulation technology is a kind of highly effective nano anti-fouling technology. It is a process in which tiny particles or droplets are surrounded by a coating to give small capsules of many useful properties. In general, it is used to incorporate food ingredients, enzymes, cells or other materials on a micro metric scale. Microencapsulation can also be used to enclose solids, liquids, or gases inside a micrometric wall made of hard or soft soluble film, in order to reduce contact of sea water and reduce the rate of release to achieve the stability of the anti-fouling effect. The micro-encapsulated anti fouling agent can obtain longer anti-fouling effect and better anti-fouling effect, and also reduce the impact to the environment. Shi et al. encapsulated non-toxic anti-fouling compound sodium benzoate in polystyrene microcapsules via an in-liquid drying process [17]. The results showed that the sodium benzoate could leach slowly from the anti-fouling coating matrix. The polymer particle size decreased while the reaction temperature deceases or amount of dispersing agent and stirring speed increasing. 


\section{Conclusion}

In this paper, traditional and recent development of anti-fouling are summarized. With the implementation of the convention on anti-fouling, to research and develop new anti-fouling materials is particularly important. At present, the marine anti-fouling coatings are developing towards the direction of high performance, energy saving, convenient construction and environmental protection. The new marine anti-fouling coatings have gradually replaced the traditional marine anti fouling coatings. Researchers pay more and more attention to non toxic self-polishing anti fouling coatings, low surface energy coatings, as well as bioactive substances containing natural bioactive substance. The new technologies such as electrolytic chemical pollution prevention and nano technology have solved the problem of low efficiency and short validity period of traditional anti-fouling coatings. In addition, composite marine anti-fouling coatings are also more and more popular. And it will be an important direction for future development.

\section{Acknowledgements}

This study was supported by the Prospective Basic Research Project of China Waterborne Transport Research Institute (WTI61421 and WTI61426).

\section{References}

[1] Coneski P. N., Weise N. K., Fulmer P. A., et al. Development and evaluation of self-polishing urethane coatings with tethered quaternary ammonium biocides [J]. Progress in Organic Coatings, 2013, 76: 1376-1386.

[2] Fabrice A., Fabienne F., Karine R, et al. Development of hybrid anti-fouling paints [J]. Progress in Organic Coatings, 2015, 87: 10-19.

[3] Lin C. H., Yeh Y. H., Lin W. C., et al. Novel silicone hydrogel based on PDMS and PEGMA for contact lens application [J]. Colloids and Surfaces B: Biointerfaces, 2014, 123: 986-994.

[4] Marceaux S., Bressy C. Development of polyorganosilazanesilicone marine coatings [J]. Progress in Organic Coatings, 2014, 77: 1919-1928.

[5] Gui T. J. Preparation and Characterization of the Organic
Silicone/Fluorine Antifouling Coatings with Low Surface Energy [D]. Ocean University of China, 2008.

[6] Qiu W. G. Investigation of marine antifouling coatings based on silicones [D]. Dalian Maritime University, 2011.

[7] Martinelli E, Suffredini M, Galli G, et al. Amphiphilic block copo-lymer/poly (dimethylsiloxane) (PDMS) blends and nanocompos-ites for improved fouling-release [J]. Biofouling, 2011, 27(5): 529-541.

[8] Chen M., Ding F., Xu L., et al. Low surface energy anti-fouling coatings based on nano- $\mathrm{SiO} 2 /$ fluorine-silicon modified acrylic resin [J]. Paint \& Coatings Industry, 2010, 2010, 40(5): 11-15.

[9] Tsoukatou M, Marechal JP, Hellio Cetal. Evaluation of the activity of the sponge metabolites avarol and avarone and their synthetic derivatives against fouling micro-and macro organisms [J]. Molecules, 2007, 12: 1022-1034.

[10] Gao M. Extraction and performance study of antifouling compounds produced by marine microorganism and microalgae [D]. Ocean University of China, 2014.

[11] Qian P. Y., Xu Y. and Fusetani N. Natural products as anti-fouling compounds: recent progress and future perspectives. Biofouling, 2010, 26(2): 223-234.

[12] Shi H. W., Liu F. C., Wang Z. Y., et al. Research Progress ofCorrosion-resisting Paints for Marine Application [J]. CORROSION SCIENCE AND PROTECTION TECHNOLOGY, 2010, 22 (1): 43-46.

[13] Xu Q, et al. Evaluation of toxicity of capsaicin and zosteric acid and their potential application as antifoulants [J]. Environ Toxicol, 2005, 20: 467-474.

[14] Fischer. Marine organism repellent covering for Protection of underwater objects and method of applying same [P]. US005226380A, 1993-07-13.

[15] Yan X. F., Yu L. M. and Jiang X. H. Synthesis of acrylamides containing capsaicin derivative and their bacteriostatic activity and antifouling capability [J]. Periodical of Ocean University of China, 2013, 43: 64-67.

[16] Fu Z. Y., Wang Q., Jia L. N., et al. The synthesis and application of isothiazolone derivativatives for recent decade [J]. Materials Review, 2012, 291-296.

[17] Shi H., Shi J. G., Chen X. L., et al. Application in marine antifouling coating [J]. Polymer Bulletin, 2011, 01: 65-70. 